\title{
Phase diagram and dynamics of Rydberg-dressed fermions in two dimensions
}

\author{
Reyhaneh Khasseh, ${ }^{1}$ Saeed H. Abedinpour, ${ }^{1,2,{ }^{*}}$ and B. Tanatar ${ }^{3}$ \\ ${ }^{1}$ Department of Physics, Institute for Advanced Studies in Basic Sciences (IASBS), Zanjan 45137-66731, Iran \\ ${ }^{2}$ Research Center for Basic Sciences \& Modern Technologies (RBST), Institute for Advanced Studies in Basic Sciences (IASBS), \\ Zanjan 45137-66731, Iran \\ ${ }^{3}$ Department of Physics, Bilkent University, 06800 Ankara, Turkey
}

(Received 5 June 2017; published 9 November 2017)

\begin{abstract}
We investigate the ground-state properties and the collective modes of a two-dimensional two-component Rydberg-dressed Fermi liquid in the dipole-blockade regime. We find instability of the homogeneous system toward phase-separated and density ordered phases, using the Hartree-Fock and random-phase approximations, respectively. The spectral weight of collective density oscillations in the homogenous phase also signals the emergence of density-wave instability. We examine the effect of exchange hole on the density-wave instability and on the collective-mode dispersion using the Hubbard local-field factor.
\end{abstract}

DOI: 10.1103/PhysRevA.96.053611

\section{INTRODUCTION}

Studying ultracold Rydberg systems is becoming a fascinating field from both fundamental and practical points of view. Such artificial systems provide a means to explore many-body models in a very controllable manner [1-7]. On the applied side, these systems are promising for the quantum manipulations and computations [8]. Different experimental methods, such as laser cooling and magnetic trapping $[9,10]$ of neutral atoms, have recently been employed. In order to reach nano-kelvin temperature regime, the magneto-optical trapping is an appropriate technique [11]. The traditional example of Rydberg atoms is hydrogen. However, any alkali-metal element can be excited to a higher-energy level, with a very high principal quantum number $n$, in which the valence electron obeys well-known Rydberg energy spectrum [12]. The effective size of these atoms can be very large, up to the micrometers [13].

Although the total charge of Rydberg atoms is zero, they can have very strong dipole moments. In this regard, there is a strong dipole-dipole interaction among Rydberg atoms. One can take advantage of this long-range interaction in order to construct strongly correlated systems. Exploring these systems seems to be very appealing and one may expect to observe some new and exotic quantum phenomena and behavior. Notice that the interaction between Rydberg atoms is very similar to the van der Waals interaction $\propto 1 / r^{6}$, at large enough interparticle distances [14-17]. However, for short inter-particle distances, called the Rydberg-blockade radius, the strong dipole-dipole interaction makes atoms off-resonant with respect to the laser frequency. As a result, the $1 / r^{6}$ van der Waals interaction of dressed atoms evolves into a soft-core interaction with finite interaction range at short distances. The typical values for this soft-core radius could reach few micrometers [18-21]. In order to construct a gas of Rydberg atoms with long lifetimes, they should be dressed, e.g., employing two laser beams where only the sum of their frequencies is on resonance with the Rydberg levels. In this way, a Rydberg atom through a two-photon absorption process

\footnotetext{
*abedinpour@iasbs.ac.ir
}

will be produced [22-24]. Exotic topological density waves in Rydberg-dressed systems in three-dimensional optical lattices has been predicted too [25].

The effect of dipole-dipole interaction results in some interesting physics. For example, density-wave instability in a three-dimensional (3D) system of Rydberg-dressed Fermi atoms, interacting through the van der Waals force, is predicted to lead to a body-centered-cubic (bcc) crystalline ordering with gapless fermionic excitations, named metallic quantum solid phase [24]. The emergence of superfluid vortices in a rotating two-dimensional (2D) Bose-Einstein condensate (BEC) of Rydberg-dressed atoms has been studied in Ref. [26]. A transition to a Bardeen-Cooper-Schrieffer (BCS) state has been also predicted in a system of Rydberg-dressed atoms under repulsive van der Waals interaction, assisted by BEC of diatomic molecules which play the analogous role of phonons in the BCS superconductivity [27].

Naturally, it is expected that the effects of many-body correlations would be enhanced at lower dimensions. Furthermore, reduced dimensionality is expected to suppress collisions and chemical reactions [28]. Motivated by these, in this paper we investigate the ground-state phases of a two-component 2D system of Rydberg-dressed atoms. Using the Hartree-Fock (HF) mean-field approach, we show that a quantum phase transition equivalent to the ferromagneticparamagnetic phase transition of an electron liquid is possible in two-component Rydberg-dressed fermionic liquid (RDL), by tuning the average density and the soft-core radius of the interaction. The term "two-component" here refers to two different fermionic isotopes of the same atom or identical atoms in two different internal states. Therefore, the abovementioned phase transition corresponds to the phase separation in the first case and to the polarization of internal degree in the later one. For brevity, in the following, we will simply refer to this phase transition as phase separation, regardless of its microscopic interpretation. Notice that the phase separation has been also predicted for fermionic systems with short-range $s$-wave scattering [29]. Moreover, we investigate the densitywave instability (DWI) of a homogenous phase-separated (i.e., single-component) RDL from the singularities of its static density-density response function within the random-phase approximation (RPA). Furthermore, we find analytical results 
for the dispersion of collective density (i.e., zero-sound) modes and the sound velocity in the phase-separated state, using the RPA. We also improve upon the RPA, including the effects of exchange-hole through the so-called Hubbard local-field factor (LFF) [30], and investigate its effects on the DWI and on the dispersion of collective mode.

The rest of this paper is organized as follows. In Sec. II we describe our model Hamiltonian and the effective interaction between Rydberg-dressed particles. In Sec. III we employ the Hartree-Fock mean-field approximation to calculate the ground-state energy of a two-component Rydberg-dressed system and investigate the possible phase transition between homogenous mixed and phase-separated states. In Sec. IV, we calculate the static dielectric function of RDL within the RPA and study the instability of the system towards the formation of density waves. We study the zero-sound mode of a homogeneous RDL using RPA in Sec. V. Finally, we summarize our main results and conclude in Sec. VI.

\section{MODEL AND HAMILTONIAN}

We consider a 2D system of two-component Rydbergdressed fermionic particles, where each particle is weakly coupled to its Rydberg state by an off-resonant two-photon transition. The Hamiltonian of this system reads

$$
\begin{aligned}
\mathcal{H}= & \sum_{\mathbf{k}, \sigma} \varepsilon_{k, \sigma} \hat{c}_{\mathbf{k}, \sigma}^{\dagger} \hat{c}_{\mathbf{k}, \sigma} \\
& +\frac{1}{2 S} \sum_{\mathbf{q}} v_{\mathbf{R}}(q) \sum_{\mathbf{k}, \sigma} \sum_{\mathbf{k}^{\prime}, \sigma^{\prime}} \hat{c}_{\mathbf{k}-\mathbf{q}, \sigma}^{\dagger} \hat{c}_{\mathbf{k}^{\prime}+\mathbf{q}, \sigma^{\prime}}^{\dagger} \hat{c}_{\mathbf{k}^{\prime}, \sigma^{\prime}} \hat{c}_{\mathbf{k}, \sigma},
\end{aligned}
$$

where $\varepsilon_{k, \sigma}=\hbar^{2} k^{2} /\left(2 m_{\sigma}\right)$ is the noninteracting energy dispersion of particles of mass $m_{\sigma}, \sigma=A, B$ refers to two different flavors of the Rydberg particles, $\hat{c}_{\mathbf{k}, \sigma}\left(\hat{c}_{\mathbf{k}, \sigma}^{\dagger}\right)$ destroys (creates) one Rydberg-dressed atom of type $\sigma$ with wave vector $\mathbf{k}$, $S$ is the sample area, and $v_{\mathrm{R}}(q)$ is the Fourier transform of the interaction between two Rydberg dressed atoms, which for simplicity we assume not to depend on the types of two atoms $\sigma$ and $\sigma^{\prime}$. Moreover, we will assume that the mass difference between two components is negligible and we set $m_{\mathrm{A}}=m_{\mathrm{B}}=m$ in all our subsequent analysis. Both of these simplifications we would expect to have only minor quantitative effects on our main findings.

In this work, we are interested in the repulsive van der Waals interaction in the far-detuning regime, i.e., $|\delta| \gg \Omega$, where $\delta<0$ and $\Omega$ are single atom red detuning and Rabi frequency, respectively. The interaction between two Rydbergdressed atoms is then dominated by the van der Waals form when the interparticle distance is large enough, i.e., $r \gg r_{c}=\left(C_{6} / 2 \hbar \delta\right)^{1 / 6}$, where $C_{6}<0$ is the bare van der Waals coefficient [31] and $r_{c}$ is the soft-core radius of the interparticle interaction. In general, the form of this interaction can be found from the perturbative expansion up to fourth order in the small parameter $\Omega /|\delta|$, which yields [32-35]

$$
v_{\mathrm{R}}(r)=\frac{D}{r^{6}+r_{c}^{6}} .
$$

Here, $r$ is the 2D distance between two atoms; $D=$ $\left|C_{6}\right|(\Omega / 2 \delta)^{4}$ is the effective van der Waals coefficient which is

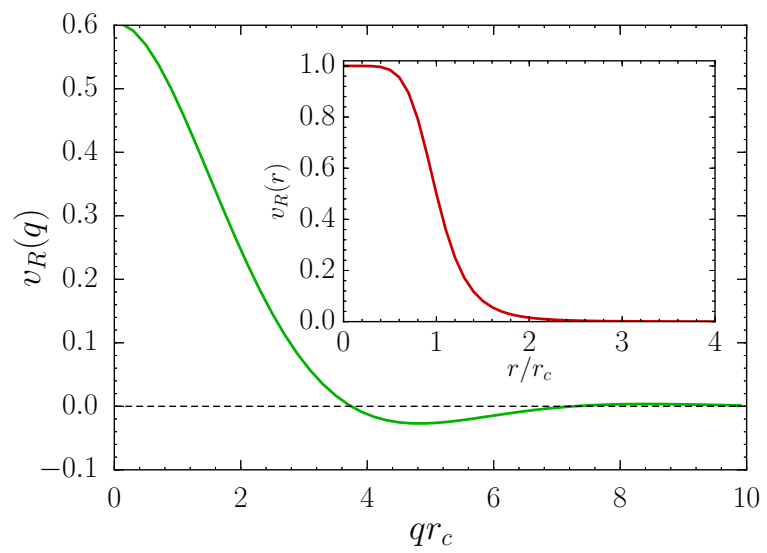

FIG. 1. Effective Rydberg-dressed potential in momentum space $v_{\mathrm{R}}(q)$ (in units of $\left.2 \pi D / r_{c}^{4}\right)$ vs $q r_{c}$. The inset shows the real-space potential $v_{\mathrm{R}}(r)$ (in units of $D / r_{c}^{6}$ ) vs $r / r_{c}$.

positive in our case, corresponding to a repulsive interparticle interaction. The Fourier transform of $v_{\mathrm{R}}(r)$ is obtained as

$$
v_{\mathrm{R}}(q)=\frac{\pi D}{3 r_{c}^{4}} G_{0,6}^{4,0}\left(\left.\frac{r_{c}^{6} q^{6}}{6^{6}}\right|_{0, \frac{1}{3}, \frac{2}{3}, \frac{2}{3}, 0, \frac{1}{3}}\right),
$$

in terms of the Meijer-G function $[36,37] G_{m n}^{i j}(\ldots)$. In Fig. 1 the behavior of interparticle interaction potential is illustrated in both position and momentum spaces. As can be seen in this figure the interaction potential in the position space is almost flat for $r<r_{c}$ and has a van der Waals tail at $r \gg r_{c}$. Although this interaction is purely repulsive in the position space, its Fourier transform $v_{\mathrm{R}}(q)$ has a negative minimum at $q \approx 5 / r_{c}$. This peculiar behavior arises from the dipole-blockade effect and is not observed in other kinds of long-range interactions [24].

Before turning to report our results, we should note that the zero-temperature properties of a $2 \mathrm{D}$ single-component fermionic RDL is characterized by two parameters, the dimensionless density parameter $\lambda=k_{\mathrm{F}} r_{0}$ and the dimensionless soft-core radius $\tilde{r}=r_{c} / r_{0}$, where $k_{\mathrm{F}}=\sqrt{4 \pi n}$ is the Fermi wave vector of a single-component system, $n$ being its particle density, and $r_{0}=\left(m D / \hbar^{2}\right)^{1 / 4}$ is the effective range of the van der Waals interaction. A two-component system requires one extra parameter specifying the density imbalance $p=$ $\left(n_{\mathrm{A}}-n_{\mathrm{B}}\right) /\left(n_{\mathrm{A}}+n_{\mathrm{B}}\right)$, where $n_{\sigma}$ is the particle density of $\sigma$ component. The density imbalance varies between $p=0$ for a two-component system with the equal population of both ingredients to $p=1$ for a single-component or fully polarized system (note that we name the higher density component as $\left.n_{\mathrm{A}}\right)$.

\section{GROUND-STATE ENERGY AND PHASE SEPARATION}

In this section, we first calculate the ground-state energy of a homogeneous RDL as a function of $\lambda, \tilde{r}$, and $p$, using the HF approximation. Then, we investigate the possibility of transition from a homogeneous and mixed phase to a separated one. The mean-field energy of a two-component system can 
be evaluated from

$$
\begin{aligned}
\langle\mathcal{H}\rangle_{\mathrm{HF}}= & \sum_{\mathbf{k}, \sigma} \varepsilon_{k} n_{k, \sigma}+\frac{1}{2 A} v_{\mathrm{R}}(q=0) \sum_{\mathbf{k}, \sigma} n_{k, \sigma} \sum_{\mathbf{k}^{\prime}, \sigma^{\prime}} n_{k^{\prime}, \sigma^{\prime}} \\
& -\frac{1}{2 A} \sum_{\mathbf{q}} v_{\mathrm{R}}(q) \sum_{\mathbf{k}, \sigma} n_{\mathbf{k}-\mathbf{q}, \sigma} n_{k, \sigma} .
\end{aligned}
$$

Here, $n_{k, \sigma}$ is the Fermi occupation function which reduces to a Heaviside step function $n_{k, \sigma}=\Theta\left(k_{\mathrm{F}, \sigma}-k\right)$ at zero temperature, where $k_{\mathrm{F}, \sigma}=\sqrt{4 \pi n_{\sigma}}$ is the Fermi wave vector of component $\sigma$. Note that in the following all the energies will be reported in the units of $E_{0}=\hbar^{2} /\left(m r_{0}^{2}\right)$. The dimensionless HF energy per particle reads

$$
\begin{aligned}
\varepsilon_{\mathrm{HF}}(p, \lambda, \tilde{r})= & \frac{\lambda^{2}}{8}\left(1+p^{2}\right)+\frac{\pi}{12 \sqrt{3}} \frac{\lambda^{2}}{\tilde{r}^{4}}-\frac{1}{2 \tilde{r}^{6}}\left[1-\frac{1}{6 \sqrt{3 \pi}}\right. \\
& \left.\times \sum_{\xi= \pm 1}(1+\xi p) g(\sqrt{1+\xi p} \lambda \tilde{r})\right]
\end{aligned}
$$

where the terms on the right-hand side are the noninteracting kinetic, Hartree, and exchange energies, respectively, and

$$
g(x)=G_{3,9}^{4,3}\left[\left.\left(\frac{x}{3 \sqrt{2}}\right)^{6}\right|_{0, \frac{1}{3}, \frac{2}{3}, 1,-\frac{1}{3}, 0, \frac{1}{3}, \frac{1}{3}, \frac{2}{3}} ^{\frac{1}{2}, \frac{5}{6}}\right] .
$$

Interestingly, both the kinetic and Hartree terms have quadratic dependence on the coupling constant $\lambda$. In Fig. 2 (a) the $\lambda$ dependence of the HF energy of a single-component (i.e., $p=1)$ 2D RDL has been illustrated. As expected, the effects of direct and exchange interactions are substantially suppressed at large soft-core radii and the system essentially becomes noninteracting. At low densities (i.e., $\lambda \rightarrow 0$ ), using the fact that

$$
g(x \rightarrow 0) \approx 3 \sqrt{3 \pi}-\frac{\sqrt{\pi^{3}}}{4} x^{2}+O\left(x^{4}\right),
$$

we can obtain the following analytic expression for the HF ground-state energy:

$$
\varepsilon_{\mathrm{HF}}(p, \lambda, \tilde{r}) \approx \frac{\lambda^{2}}{8}\left(1+p^{2}\right)+\frac{\pi}{24 \sqrt{3}} \frac{\lambda^{2}}{\tilde{r}^{4}}\left(1-p^{2}\right)+O\left(\lambda^{4}\right),
$$

where the second term corresponds to the sum of Hartree and the leading-order contribution from exchange energy to the ground state. According to the above expression, in a single-component system (i.e., $p=1$ ), the Hartree term is exactly canceled by the leading-order contribution from exchange energy, while in an unpolarized system (i.e., $p=0$ ), only half of the Hartree energy would be canceled by exchange. Further inspection of Eq. (5) for $p=0$ and $p=1$ reveals a quantum phase transition between unpolarized and fully polarized phases as functions of particle density and soft-core radius parameter $r_{c}$ [see Fig. 2(b)]. This is totally equivalent to the paramagnetic-ferromagnetic phase transition in the electron liquid [30]. We have also verified that the energy of a partially polarized state is always higher than either the mixed (i.e., unpolarized) or fully separated state. Therefore, it never becomes the ground state of a two-component system. (a)

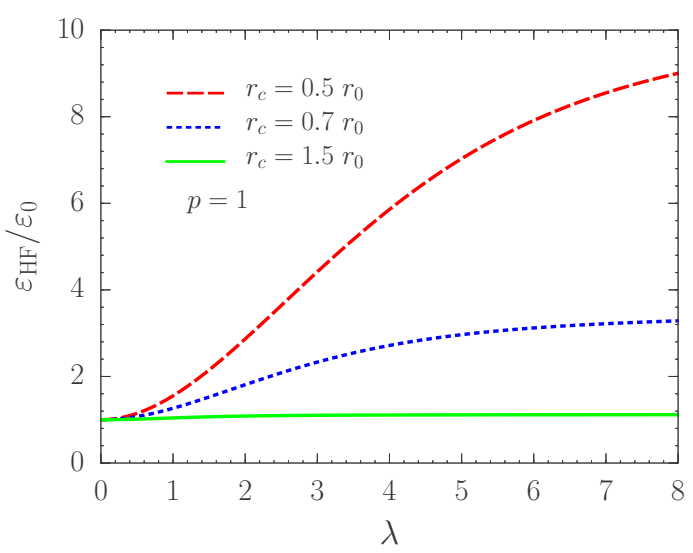

(b)

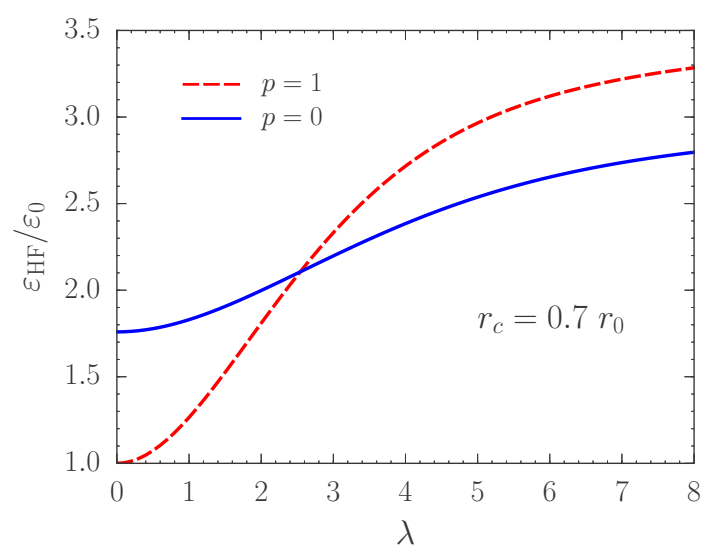

FIG. 2. (a) Ground-state energy of a single-component 2D Rydberg-dressed liquid within the HF approximation (in units of the noninteracting ground-state energy $\varepsilon_{0}=E_{0} \lambda^{2} / 4$ of a singlecomponent system) vs the coupling strength $\lambda$ for several values of $r_{c} / r_{0}$. (b) Comparison of the HF energies of mixed and phaseseparated states (in units of $E_{0} \lambda^{2} / 4$ ) vs $\lambda$ for $r_{s}=0.7 r_{0}$. The phase-separated state becomes lower in energy for $\lambda \lesssim 2.5$.

The phase diagram of mixed and phase-separated states is shown in Fig. 3 in the $\lambda-\tilde{r}$ parameter space. As it is seen in the figure, for low-density and small soft-core radius the system is in the phase-separated state.

In the remaining parts of this work, we will discuss the density stability and collective excitations of the phase-separated ground state, which can be regarded as a single-component RDL.

\section{DENSITY-WAVE INSTABILITY OF THE GROUND STATE}

In this section, employing the random-phase approximation, we investigate the DWI of a single-component RDL from the poles of its static density-density response function, or equivalently from the zeros of its static dielectric function

$$
\epsilon_{\mathrm{RPA}}(q)=1-v_{\mathrm{R}}(q) \chi_{0}(q)=0,
$$

where $v_{\mathrm{R}}(q)$ is given in Eq. (3) and $\chi_{0}(q)$ stands for the noninteracting static density-density response function of a single-component 2D Fermi gas with parabolic energy 


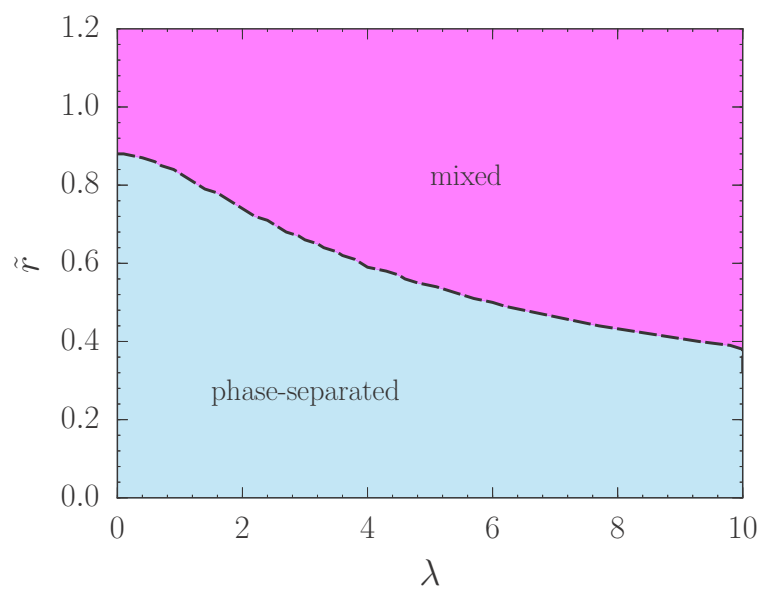

FIG. 3. Competition between mixed and phase-separated states of a two-dimensional two-component Rydberg-dressed system in the $\lambda-\tilde{r}$ parameter space, obtained within the Hartree-Fock approximation.

dispersion [30]

$$
\chi_{0}(q)=-v_{0}\left[1-\Theta\left(q-2 k_{\mathrm{F}}\right) \frac{\sqrt{q^{2}-4 k_{\mathrm{F}}^{2}}}{q}\right],
$$

where $\nu_{0}=m /\left(2 \pi \hbar^{2}\right)$ is the density of states of a singlecomponent 2D liquid. For a given set of system parameters, i.e., $\lambda$ and $r_{c} / r_{0}$, if the static dielectric function becomes zero at a specific wave vector $q_{I}$, the homogenous system becomes unstable towards a density ordered phase with wavelength $\lambda_{I}=2 \pi / q_{I}$. However, it should be noted that as this instability criterion corresponds to a second-order phase transition from a homogeneous into an inhomogeneous state, a first-order phase transition of the homogenous state might precede this instability, in which case no instability would emerge [38-40]. Note that as the static response function (10) itself is negative, the density-wave instability would be feasible only in the negative regions of the interparticle interaction i.e., $q_{I} \approx 5 / r_{c}$ within the RPA. In other words, the magnitude of the instability wave vector is determined by the soft-core radius of the interaction. If this wave vector becomes larger than $\sim 2 k_{\mathrm{F}}$, the wavelength of the expected density ordered phase will be smaller than the average interparticle separation. Therefore, in order to determine DWI, we should search for the solutions of Eq. (9) with $q \leqslant 2 k_{\text {F. }}$. The phase boundary between stable homogeneous and the density-wave phases obtained within the RPA, and with the condition $q_{I} \leqslant 2 k_{\mathrm{F}}$, is illustrated in Fig. 4 .

In the RPA, the effects of exchange and correlation are completely absent. Improvements over RPA could be achieved by replacing the bare interaction $v_{\mathrm{R}}(q)$ in Eq. (9) with an effective one

$$
v_{\text {eff }}(q)=v_{\mathrm{R}}(q)[1-G(q)],
$$

where $G(q)$ is the local-field factor [30]. Using the Hubbard approximation for the $\operatorname{LFF}_{\mathrm{H}}(q)=v_{\mathrm{R}}\left(\sqrt{k_{\mathrm{F}}^{2}+q^{2}}\right) / v_{\mathrm{R}}(q)$, we have reexamined the DWI, and the corresponding result is shown by dotted red lines in Fig. 4. Evidently, the homogenous

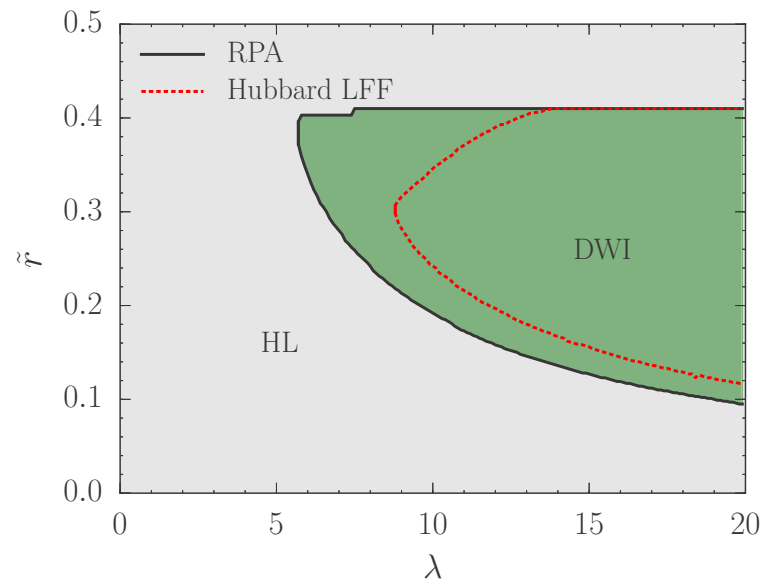

FIG. 4. Regions of stable homogeneous liquid (HL) and the density-wave instability (DWI) of a single-component twodimensional Rydberg-dressed liquid in the $\lambda-\tilde{r}$ plane. Black solid lines show the phase boundary within the random-phase approximation, and red dotted lines refer to the phase boundary obtained using the Hubbard approximation for local-field factor. Within both approximations, only the unstable regions with $q_{I} \leqslant 2 k_{\mathrm{F}}$ for the instability wave vector have been retained.

liquid phase becomes more stable when the effects of exchange hole are included through the Hubbard LFF.

\section{ZERO-SOUND MODES}

The collective density oscillations of an interacting system could be obtained from the zeros of its dynamic dielectric function. In the RPA, one should look for the solutions of

$$
1-v_{\mathrm{R}}(q) \operatorname{Re} \chi_{0}(q, \omega)=0,
$$

outside the particle-hole continuum (PHC), i.e., in the regions of frequency-wave vector plane where the imaginary part of the noninteracting density response function vanishes $\operatorname{Im} \chi_{0}(\boldsymbol{q}, \omega)=0$.

Using the analytic forms of the real and imaginary parts of the noninteracting density response function of a twodimensional Fermi system [30], it turns out that it is possible to find an analytic solution for Eq. (12) outside the PHC, which gives the full dispersion of the zero-sound mode as

$$
\begin{aligned}
\omega_{\mathrm{ZS}}(q)= & v_{\mathrm{F}} q\left[1+\frac{1}{v_{0} v_{\mathrm{R}}(q)}\right] \\
& \times \sqrt{\left(\frac{q}{2 k_{\mathrm{F}}}\right)^{2}+\frac{v_{0}^{2} v_{\mathrm{R}}^{2}(q)}{1+2 v_{0} v_{\mathrm{R}}(q)}},
\end{aligned}
$$

with $v_{\mathrm{F}}=\hbar k_{\mathrm{F}} / m$ being the Fermi velocity.

In the long-wavelength limit, we have $v_{\mathrm{R}}(q \rightarrow 0) \approx u_{0}=$ $\pi^{2} D /\left(3 \sqrt{3} r_{c}^{4}\right)$ and, by plugging it in Eq. (13), we find $\omega_{\mathrm{ZS}}(q \rightarrow 0) \approx v_{\mathrm{ZS}} q$, where

$$
v_{\mathrm{ZS}}=\frac{1+v_{0} u_{0}}{\sqrt{1+2 v_{0} u_{0}}} v_{\mathrm{F}}
$$

is the sound velocity. Evidently, this velocity is larger than $v_{\mathrm{F}}$; therefore, the zero-sound wave is undamped at long wavelengths. Effects of exchange-correlation hole in the 
(a)

(b)
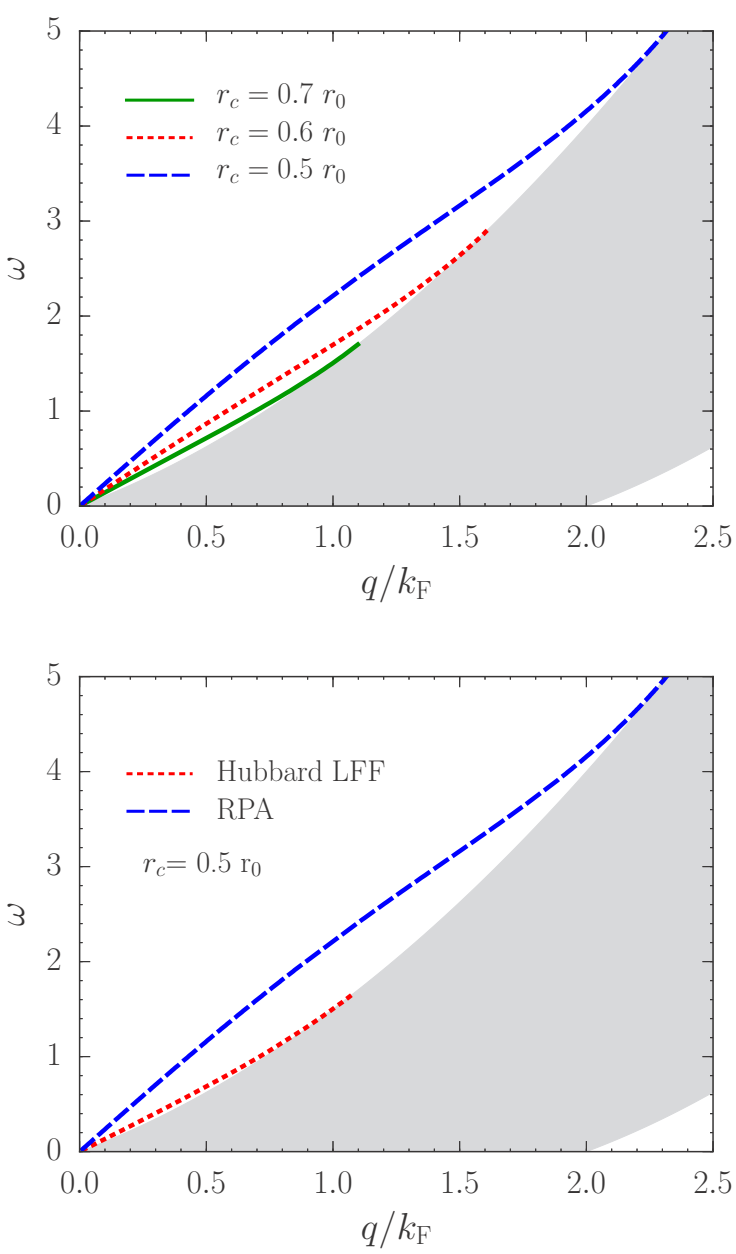

FIG. 5. (a) Dispersion of the zero-sound mode of a singlecomponent 2D Rydberg-dressed liquid within the RPA (in units of $\left.\hbar k_{\mathrm{F}}^{2} / m\right)$ vs $q / k_{\mathrm{F}}$ for several values of $r_{c} / r_{0}$. (b) Effects of the Hubbard LFF on the dispersion of zero-sound for $\lambda=2$ and $r_{c}=0.5 r_{0}$. Filled areas in gray color indicate the particle-hole continuum.

dispersion of zero sound could be obtained by replacing the bare interaction $v_{\mathrm{R}}(q)$ in Eq. (13) with the effective interaction of Eq. (11). In Fig. 5(a) the full dispersion of the zero-sound mode within the RPA is plotted for different values of the soft-core radius $r_{c}$. In Fig. 5(b) the effects of Hubbard LFF on the dispersion of zero sound is depicted. Notice that exchange hole substantially suppresses the zero-sound velocity.

We also investigate the oscillation strength, or the spectral weight of the collective mode, from the imaginary part of the RPA density response function

$$
\operatorname{Im} \chi^{\mathrm{RPA}}(q, \omega)=\frac{\operatorname{Im} \chi_{0}(q, \omega)}{\left|1-v_{\mathrm{R}}(q) \chi_{0}(q, \omega)\right|^{2}} .
$$

This spectral weight has a Dirac delta peak over the collectivemode dispersion outside the PHC and has contributions from both single particle and collective excitations inside the continuum. In Fig. 6, the spectral weight has been depicted versus frequency and wave vector. For the density and soft-core radius values, where the DWI is expected, mode softening and
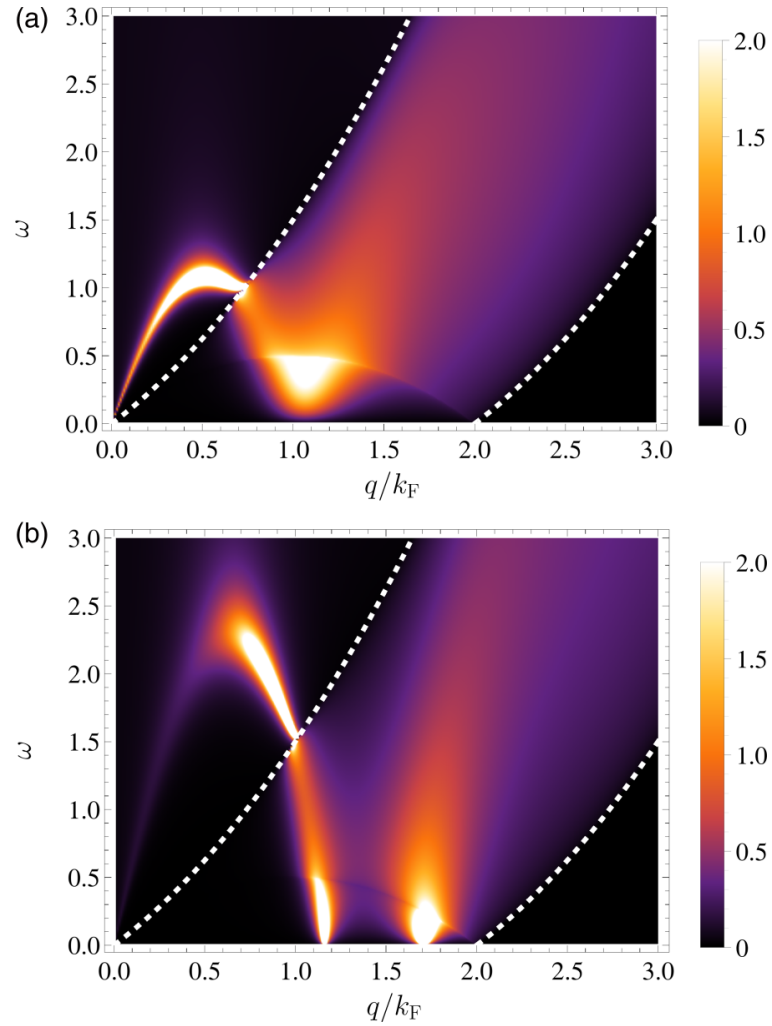

FIG. 6. Imaginary part of the density response function within the RPA vs $\omega$ (in units of $\hbar k_{\mathrm{F}}^{2} / m$ ) and $q$ (in units of $k_{\mathrm{F}}$ ) at a fixed density parameter $\lambda=10$, and for $\tilde{r}=0.45$ (a) and $\tilde{r}=0.3$ (b). These two parameter values for $\tilde{r}$ respectively correspond to the homogenous liquid and density-wave unstable regions of the phase diagram (see Fig. 4). Note that the Dirac delta peak of the spectral weight outside the PHC has been broadened by $\sim 0.01$ for a better visibility.

a roton-like minimum, which reaches zero at $q \sim q_{I}$, is evident in the spectral weight (see the lower panel of Fig. 6).

\section{SUMMARY AND CONCLUSIONS}

In this paper, we have studied the ground-state phases of a two-dimensional Rydberg-dressed Fermi liquid. The physics of this system is governed by the competition between three natural length scales of the system, namely the soft-core radius $r_{c}$, the range of effective van der Waals interaction $r_{0}$, and the average distance between atoms $1 / \sqrt{n}$. In fact, the Rydbergdressed interaction has its strongest repulsive effects for $r_{c} \lesssim$ $r \lesssim r_{0}$. This well explains our Hartree-Fock prediction for the phase separation in a two-component system at small soft-core radius and at low densities, where the repulsive interaction of each particle with its nearest neighbors is dominant. At larger soft-core radii or higher densities, the nearest neighbors of each particle will fall within its soft core and will be affected only by a very weak repulsive force.

In a single-component system, we have looked for the signatures of the instability of a homogeneous system towards density ordered phases from the singularities of its static dielectric function. The DWI is expected for high densities and at intermediate $r_{c}$ values. At large soft-core radii, the system is effectively noninteracting, while at intermediate values of 
$r_{c}$, particles would energetically prefer to aggregate together within the soft core of each other and increase the average distance between clusters of particles. Therefore, inhomogeneous density phases such as density waves or quantum droplets [41] would be naturally expected in this regime. Here, we should mention that the density-wave instability within different approximations has been also predicted for ultracold dipolar systems with anisotropic dipole-dipole interaction [42-45], as well as in layered dipolar structures [46-48]. However, its emergence in a single-layer system, with a purely isotropic dipole-dipole interaction, has been the subject of much dispute $[49,50]$. Here, we have shown that such an instability could be anticipated in a single layer of Rydberg-dressed atoms with isotropic interaction. Also, we have examined that this instability is not an artifact of the random phase approximation, and it survives when the effects of exchange hole have been also taken into account.

We have also obtained the full analytic dispersion of collective density oscillations in a single-component Rydbergdressed Fermi liquid. This dispersion is undamped and linear at long wavelengths. Similar to the three-dimensional Rydberg-dressed liquids [24], mode softening in the vicinity of density instability has been observed in the spectral weight of collective mode.

Experimentally, one can start from a weakly interacting gas of Fermi atoms trapped inside a pancakelike quasi-two- dimensional trap and then excite a small fraction of atoms into Rydberg states. For example, if we take ${ }^{40} \mathrm{~K}$ atoms excited to the $62 S$ state, the bare van der Waals coefficient would be $C_{6} / \hbar \approx 2 \pi \times 129.8 \mathrm{GHz} \mu \mathrm{m}^{6}$ [31]. With the laser detuning of $|\delta|=2 \pi \times 2 \mathrm{MHz}$, the soft-core radius becomes $r_{c} \approx$ $5.6 \mu \mathrm{m}$. If the Rabi frequency $\Omega=2 \pi \times 440 \mathrm{kHz}$ is chosen, the effective interaction range of $r_{0} \approx 16.5 \mu \mathrm{m}$ could be obtained. The interesting phenomena such as phase separation and density wave instability predicted in this work require a relatively low planar density of $\sim 10^{6}-10^{7} \mathrm{~cm}^{-2}$ for Rydberg atoms. The main challenge though seems to be the finite lifetime of Rydberg states. However, the effective decay rate of dressed states $\gamma_{\text {eff }}=(\Omega / 2 \delta)^{2} \gamma$ are substantially reduced with respect to the natural decay rate of bare states $\gamma$, resulting in typical lifetimes of few hundreds of milliseconds for Rydbergdressed atoms [32]. We would expect this effective lifetime to be long enough to make the experimental observation of different phases predicted here feasible.

\section{ACKNOWLEDGMENTS}

We thank Habib Rostami and Sebastian Wüster for useful comments and suggestions. This work is supported in part by Turkish Academy of Sciences (TUBA). S.H.A. is grateful for the hospitality of Physics Department at Bilkent University during the final stages of this work.
[1] P. Schauß, J. Zeiher, T. Fukuhara, S. Hild, M. Cheneau, T. Macrí, T. Pohl, I. Bloch, and C. Gross, Science 347, 1455 (2015).

[2] J. Zeiher, J. Choi, A. Rubio-Abadal, T. Pohl, R. van Bijnen, I. Bloch, and C. Gross, arXiv:1705.08372.

[3] J. Zeiher, P. Schauß, S. Hild, T. Macrí, I. Bloch, and C. Gross, Phys. Rev. X 5, 031015 (2015).

[4] A. W. Glaetzle, M. Dalmonte, R. Nath, C. Gross, I. Bloch, and P. Zoller, Phys. Rev. Lett. 114, 173002 (2015).

[5] J. Zeiher, R. van Bijnen, P. Schauß, S. Hild, J. Choi, T. Pohl, I. Bloch, and C. Gross, Nat. Phys. 12, 1095 (2016).

[6] H. Labuhn, D. Barredo, S. Ravets, S. de Léséleuc, T. Macrí T. Lahaye, and A. Browaeys, Nature (London) 534, 667 (2016).

[7] M. Marcuzzi, J. Minář, D. Barredo, S. de Léséleuc, H. Labuhn, T. Lahaye, A. Browaeys, E. Levi, and I. Lesanovsky, Phys. Rev. Lett. 118, 063606 (2017).

[8] M. D. Lukin, M. Fleischhauer, R. Cote, L. M. Duan, D. Jaksch, J. I. Cirac, and P. Zoller, Phys. Rev. Lett. 87, 037901 (2001).

[9] H. J. Metcalf and P. V. D Straten, Laser Cooling and Trapping (Springer-Verlag, New York, 2012).

[10] W. D. Phillips, Rev. Mod. Phys. 70, 721 (1998).

[11] J. M. Choi, J. M. Kim, S. Y. Jeong, and D. Cho, J. Korean Phys. Soc. 46, 425 (2005).

[12] D. Kleppner, M. G. Littman, and M. L. Zimmerman, Sci. Am. 244, 130 (1981).

[13] C. Fabre, M. Gross, J. M. Raimond, and S. Haroche, J. Phys. B: At. Mol. Phys. 16, L671 (1983).

[14] J. Lim, H. Lee, and J. Ahn, J. Korean Phys. Soc. 63, 867 (2013).

[15] A. Browaeys and T. Lahaye, in Niels Bohr, 1913-2013: Poincarè Seminar 2013, edited by O. Darrigol, B. Duplantier, J. M. Raimond, and V. Rivasseau (Springer, Switzerland, 2016), p. 177.
[16] R. Faoro, B. Pelle, and A. Zuliani, Eur. Phys. J. Spec. Top. 225, 2935 (2016).

[17] T. Lahaye, T. Koch, B. Fröhlich, M. Fattori, J. Metz, A. Griesmaier, S. Giovanazzi, and T. Pfau, Nature (London) 448, 672 (2007).

[18] D. Comparat and P. Pillet, J. Opt. Soc. Am. B 27, A208 (2010).

[19] M. Weidemüller, Nat. Phys. 5, 91 (2009).

[20] D. Tong, S. M. Farooqi, J. Stanojevic, S. Krishnan, Y. P. Zhang, R. Côté, E. E. Eyler, and P. L. Gould, Phys. Rev. Lett. 93, 063001 (2004).

[21] K. Singer, M. Reetz-Lamour, T. Amthor, L. G. Marcassa, and M. Weidemüller, Phys. Rev. Lett. 93, 163001 (2004).

[22] S. Wüster, C. Ates, A. Eisfeld, and J. M. Rost, New J. Phys. 13, 073044 (2011).

[23] B. Balewski, A. T. Krupp, A. Gaj, S. Hofferberth, R. Löw, and T. Pfau, New J. Phys. 16, 063012 (2014).

[24] W.-H. Li, T.-C. Hsieh, C.-Y. Mou, and D.-W. Wang, Phys. Rev. Lett. 117, 035301 (2016).

[25] X. Li and S. Das Sarma, Nat. Commun. 6, 7137 (2015).

[26] N. Henkel, F. Cinti, P. Jain, G. Pupillo, and T. Pohl, Phys. Rev. Lett. 108, 265301 (2012).

[27] T. Espinosa-Ortega and I. A. Shelykh, arXiv:1606.01664.

[28] M. A. Baranov, M. Dalmonte, G. Pupillo, and P. Zoller, Chem. Rev. 112, 5012 (2012).

[29] E. Fratini and S. Pilati, Phys. Rev. A 90, 023605 (2014).

[30] G. F. Giuliani and G. Vignale, Quantum Theory of the Electron Liquid (Cambridge University Press, Cambridge, UK, 2005).

[31] K. Singer, J. Stanojevic, M. Weidemüller, and R. Côté, J. Phys. B 38, S295 (2005). 
[32] N. Henkel, R. Nath, and T. Pohl, Phys. Rev. Lett. 104, 195302 (2010).

[33] J. Honer, H. Weimer, T. Pfau, and H. P. Büchler, Phys. Rev. Lett. 105, 160404 (2010).

[34] B. Xiong, H. H. Jen, and D.-W. Wang, Phys. Rev. A 90, 013631 (2014).

[35] J. E. Johnson and S. L. Rolston, Phys. Rev. A 82, 033412 (2010).

[36] I. S. Gradstein and I. M. Ryzhik, Table of Integrals, Series, and Products, 8th ed. (Academic Press, New York, 2014).

[37] E. W. Weisstein, Meijer G-Function, from MathWorld-A Wolfram Web Resource, http://mathworld.wolfram.com/MeijerGFunction.html.

[38] D. Neilson, L. Świerkowski, J. Szymański, and L. Liu, Phys. Rev. Lett. 71, 4035 (1993).

[39] J. Szymański, L. Świerkowski, and D. Neilson, Phys. Rev. B 50, 11002 (1994).

[40] L. Liu, L. Świerkowski, D. Neilson, and J. Szymański, Phys. Rev. B 53, 7923 (1996).
[41] F. Cinti, P. Jain, M. Boninsegni, A. Micheli, P. Zoller, and G. Pupillo, Phys. Rev. Lett. 105, 135301 (2010).

[42] Y. Yamaguchi, T. Sogo, T. Ito, and T. Miyakawa, Phys. Rev. A 82, 013643 (2010).

[43] K. Sun, C. Wu, and S. Das Sarma, Phys. Rev. B 82, 075105 (2010).

[44] M. M. Parish and F. M. Marchetti, Phys. Rev. Lett. 108, 145304 (2012).

[45] B. P. van Zyl, W. Kirkby, and W. Ferguson, Phys. Rev. A 92 , 023614 (2015).

[46] E. Akaturk, B. Tanatar, and S. H. Abedinpour, arXiv:1507.06185.

[47] J. K. Block, N. T. Zinner, and G. M. Bruun, New J. Phys. 14, 105006 (2012).

[48] F. M. Marchetti and M. M. Parish, Phys. Rev. B 87, 045110 (2013).

[49] S. H. Abedinpour, R. Asgari, B. Tanatar, and M. Polini, Ann. Phys. (NY) 340, 25 (2014).

[50] N. Matveeva and S. Giorgini, Phys. Rev. Lett. 109, 200401 (2012). 